\title{
Chronos - take the pulse of our galactic neighbourhood
}

\section{After Gaia: Time domain information, masses and ages for stars}

\author{
Eric Michel, et al. [full author details at the end of the article]
}

Received: 27 July 2020 / Accepted: 15 March 2021 / Published online: 31 July 2021

(C) The Author(s) 2021

\begin{abstract}
Understanding our Galaxy's structure, formation, and evolution will, over the next decades, continue to benefit from the wonderful large survey by Gaia, for astrometric, kinematic, and spectroscopic characterization, and by large spectroscopic surveys for chemical characterization. The weak link for full exploitation of these data is age characterization, and stellar age estimation relies predominantly on mass estimates. The ideas presented in this White Paper shows that a seismology survey is the way out of this situation and a natural complement to existing and planned surveys. These ideas are strongly rooted in the past decade's experience of the so-called Seismology revolution, initiated with CoRoT and Kepler. The case of red giant stars is used here as the best current illustration of what we can expect from seismology for large samples, but premises for similar developments exist in various other classes of stars covering other ranges of age or mass. Whatever the star considered, the first information provided by stellar pulsations is always related to the mean density and thus to the mass (and age). In order to satisfy the need for long-duration and allsky coverage, we rely on a new instrumental concept which decouples integration time and sampling time. We thus propose a long ( 1 year) all-sky survey which would perfectly fit between TESS, PLATO, and the Rubin Observatory (previously known as LSST) surveys to offer a time domain complement to the current and planned astrometric and spectroscopic surveys. The fine characterization of host stars is also a key aspect for the interpretation and exploitation of the various projects - anticipated in the framework of the Voyage 2050 programme - searching for atmospheric characterization of terrestrial planets or, more specifically, looking for a signature of life, in distant planets.
\end{abstract}

Keywords Milky way galaxy · Stellar ages · Time domain astrophysics $\cdot$ Seismology

\section{Introduction - understanding our galaxy with Gaia and time domain constraints}

Galactic archaeology has entered a new era with the data provided by the Gaia mission, providing new insights into fundamental questions about the build-up of our Galaxy 
and its different stellar components. For instance, is the actual backbone of our Galaxy a "disky" stellar component, or was there an early in-situ stellar halo? What is the role played by early mergers and accretions of satellite galaxies? What was (and still currently is) the respective role of external accretions and internal dynamical instabilities (bar, spiral arms), as well as their interplays, in shaping the star formation history and the dynamics of the different stellar components? Answering all these questions requires determining the ages of stars.

The Gaia mission [1] is providing us with an unprecedented insight into our Galaxy. Completed by the associated large spectroscopic surveys (such as APOGEE and WEAVE), Gaia is characterizing in detail and with a unique precision, positions (including distances), proper motions, and magnitudes for about a billion stars, and 3D motions, effective temperatures, and chemical compositions for several tens of millions of stars over a large part of our Galaxy and nearby satellite galaxies (e.g., [2]).

These precise data shed a new light on the above fundamental questions about how our Galaxy has formed and evolved. They reveal unexpected trends, suggesting for instance that a large fraction of the oldest stars in the Galaxy have "disky" orbits [3], that the stellar halo is almost fully accreted (e.g., [4]) and that these accreted components in turn played an important role in shaping the in-situ "disky" components (e.g., [5]), while it appears that more recent accretions are still influencing disc dynamics today $[6,7]$. The respective roles of such external perturbations and internal instabilities such as the Galactic bar, the structure of which is also being constrained with Gaia (e.g., $[8,9]$ ), and the interplay between these external and internal dynamical mechanisms, are however still under investigation. To reach a more precise view of the situation, it is essential to enrich the very precise astrometric-kinematic-chemical view of the Galaxy provided by Gaia and large spectroscopic surveys with high precision ages which can be provided through seismic information (or more generally time domain information).

A large fraction of stars show multi-periodic photometric variations due to stellar pulsations, as well as, for example, rotational modulation or granulation. These phenomena and the measurement of their photometric signature carry valuable information of a different nature to the astrometric-kinetic-chemical one, like mass, age and (unprojected) rotation estimates. With CoRoT [10] and Kepler [11], we have learned to derive this information from photometric light curves of tens of thousands of stars and we can foresee an upgrade of this capacity in the number of stars but also in type of stars and in type of applications.

This is why we are firmly convinced that it will be a top priority for the scientific community in 2035-50 to improve our view and understanding of our Galaxy, by adding a time domain dimension to the Gaia Survey. Chronos will complete the Gaia survey with high-temporal resolution for a million stars with $8 \leq m_{V} \leq 11$.

This paper is organised as follows:

- $\quad$ in Section 2 we discuss the nature of the information brought by the variability dimension and show why it is original and complementary to information on stars brought by Gaia and spectroscopic surveys and why a strong potential synergy exists. We also stress the specific constraints characterizing time domain measurements with a special emphasis on the project considered here. 
- in Section 3 we show how our understanding of the Galaxy will progress with the variability information. In order to be practical, we realise this study in the framework of a specific example of survey focussing on red giants for which an instrumental option is proposed in the next Section.

- $\quad$ in Section 4 we describe a possible option for an instrument and a mission profile capable of providing such a variability survey. We compare this instrument with existing or planned missions and show how unique and well suited it is for our purpose.

- Section 5 addresses possible improvements for the scientific objective and technical challenges for the mission concept presented here.

- in Section 6 we summarize our proposal and conclude with a broad scientific perspective.

\section{Specific nature of time domain characterization and synergy with Gaia and large spectroscopic surveys}

As already mentioned, photometric variability exists at different amplitude levels and different time scales in a large proportion of stars. This variability can be due to stellar pulsations, but also to other phenomena like rotation modulation associated with activity or binary tide effects, granulation, to name a few.

\subsection{Specificity of time domain information}

Considering stellar pulsation and seismic information, the case of red giant stars is very illustrative of the power of this approach. From the observation of several tens of thousands of red giants by CoRoT and Kepler, we have learned to understand their oscillation spectra and to interpret them in terms of a few parameters, the so-called seismic indices (e.g. [12-18]):

- $\Delta v$ : known as the large separation, characterizes the first-order regularity in frequency of acoustic oscillation modes. This seismic index is tightly linked with the stellar mean density [19]. It is a very valuable piece of information about the individual stars, because, via the strong relation between radius and mass it imposes, it brings the possibility to characterize the stars in terms of mass, which is not measured with Gaia and only very poorly (via $\log g$ ) in the spectroscopic analysis.

- $\quad v_{\max }$ : the frequency of maximum pulsation amplitude is strongly linked with the surface gravity, and, to a lesser extent with the effective temperature [20]. It is another very valuable piece of information about the individual stars, because it gives accurate $\log g$ with a precision much better than spectroscopic analysis. When spectroscopic estimates of $\log g$ are known within a factor 2 or 3 for field stars, so-called 'seismic $\log g$ ' are measured with a precision better than $2 \%$ [21].

The seismic indices $\Delta v$ and $v_{\max }$, combined with the spectroscopic measurements of effective temperature, are used to provide stellar masses and radii based on simple seismic scaling relations. They have been used to derive precise estimates of individual 
radii and masses for about 55,000 red giant stars observed with CoRoT $(\sim 10,000)$, Kepler $(\sim 20,000)$, K2 $(\sim 10,000)$, and TESS $(\sim 15,000)$ (e.g. [22-24]). These seismic indices are available online (see e.g. the Stellar Seismic Indices data base http://ssi.lesia. obspm.fr/). They are also regularly used in an iterative spectroscopic analysis to produce more precise gravity estimates allowing more precise chemical composition analysis.

For several thousands of these red giants, with long enough observations, it has been possible to measure a third seismic index [25]:

- $\Delta \mathrm{P}$ is the period spacing for g-dominated oscillation dipole modes. Its asymptotic value measures the size of the inner radiative core of red giants [26]. It is thus a very precise proxy of the evolution of the stellar structure with age, even if measured in non-asymptotic conditions [27]. It allows for instance a clear distinction between a horizontal branch red giant (or red clump, RC here after) having ignited the fusion of helium in a small convective core and a red giant branch star still having an inert helium core [12] although both stars might have exactly the same radius and effective temperature.

The situation is still progressing in order to characterize both the core and the envelope of the red giants. Rotation of the red giant core can be measured [16, 17, 28, 29]. Recently, Géhan et al. [30] could perform the automatic measurement of the rotation for about 900 red giants on the RGB. Several works are under way to make automatic and extend to a large number of stars the measurements of the pseudo-periodic perturbation of the pulsation frequency pattern characterizing the acoustic depth of the outer convective zone [31]; in parallel, further information on the convection comes from the measurement of the mode lifetimes [32] and of the luminosity bump [33].

The potential of asteroseismology illustrated above on red giants can be extended to a large number and variety of stars. The measurement and use of $\Delta v$ and $v_{\max }$, for instance, can be straightforwardly applied to other solar-type pulsators (stars showing, as the Sun and red giants, pulsations excited by the near surface convection) which cover a large part of the Hertzsprung-Russell (HR) diagram. This includes all main sequence stars cooler than early F, subgiants, and AGB stars.

Beyond solar-type oscillations many classical pulsators spanning various mass ranges and evolution stages in the HR diagram provide in their pulsation spectra patterns like $\Delta v$ or individual eigenfrequency values indicative of the mean star density. This is well known for Cepheids and RR Lyrae, but it is also potentially the case for main sequence pulsators of different mass. For instance, $\delta$ Scuti stars are 1.5-2.5 M stars in the main sequence and early subgiant phase. The analysis of their oscillation based on about 1800 of them observed with CoRoT has confirmed the existence of a regular pattern [34-37] and this frequency spacing, very similar to $\Delta v$ has been shown to be a precise indicator of the mean density [38, 39]. The effective extension of the seismic characterization is thus to a large extent a matter of collecting precise photometric time series of large enough samples of these various pulsators in order to achieve the comprehensive analysis of their pulsation spectra. 
Rotation and activity Several thousands of light-curves obtained with CoRoT and Kepler have revealed the signature of rotational modulation attributed to starspots [40-44]. In addition to providing a measurement of the rotational period free of the sin $i$ projection factor, these light-curves revealed that the signature of activity is not only found in late F-G stars, for which magnetic fields and starspots were expected as in the Sun, but also in intermediate A and B type stars [45-47]. This interpretation is supported by the discovery of the weak signature of magnetic field in A and B type stars [48-50]. Since the surface rotation rate of a star is in the first place a function of its mass and evolutionary state (at least for low-mass stars), gyrochronology (e.g. [51, 52]) takes advantage of these measurements of the stellar rotation rate to produce an estimate of age.

Granulation The light-curves of red giants have also revealed the presence of a signal characterizing the stellar granulation, which is a superficial signature of convection inside the stellar envelope. As for the seismic indices, it is possible to extract in a rather easy way the characteristic parameters of stellar granulation. These parameters also obey scaling relations, which in addition to seismic scaling relations, provide information about the surface layers of the star. In particular, the CoRoT and Kepler observations have revealed the existence of a universal scaling relation between the characteristic time scale of stellar granulation and surface gravity $[14,53-55]$; in the absence of detectable oscillations, this scaling provides an estimate of a star's surface gravity.

\subsection{Specific observational constraints}

A practical difficulty exists however to design a unique large sky coverage survey for seismic characterization of stars in our Galaxy. It resides in the different pulsation time scales and amplitudes between these evolution stages. The main sequence objects for instance are the more demanding in noise level, with amplitudes of the order of $1 \mathrm{ppm}$ $\mu \mathrm{Hz}^{-1 / 2}$ and in sampling cadence ( $\sim 1$ min or less), but a couple of months are enough to resolve their eigenfrequency pattern and extract seismic indices. At the other end of the

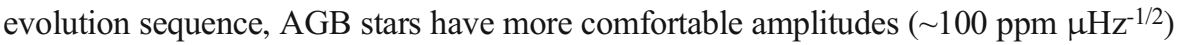
and sampling cadence (a few days are sufficient) but the frequency resolution necessary to measure their large separation $\Delta v$ and $v_{\max }$ requires long durations, of the order of 7 to 10 years (see [56-59]).

This is why it is reasonable to plan a few distinct surveys, each optimized for a given domain of the HR diagram.

As we will show in Section 4, PLATO represents presently the best opportunity to lead a substantial survey on main sequence solar-type pulsators on about half the sky. At the other extremity of the HR diagram, the Rubin Observatory is very promising for a survey of AGB stars on about half the sky too.

In the present White Paper, we consider the intermediate domain between these two extremes. We focus on what we can learn about our Galaxy with a survey able to characterize seismically all stars from subgiants, to red giants and early AGB stars in a few kpc around our Sun. 


\section{How our understanding of our galaxy will increase with a fast cadence, long duration variability survey of red giant stars over a few kpc}

The understanding of our Galactic surrounding and of our Galaxy as a whole is experiencing unprecedented progress under the influence of the Gaia mission [1] combined with ongoing and soon-to-start large spectroscopic surveys, such as APOGEE [60], WEAVE [61] or 4MOST [62]. In this context and in the 2035-50 perspective, we illustrate how this understanding can be dramatically improved with information about mass, age, rotation, etc.... brought by a variability survey [63-68].

In order to illustrate in a practical way the interest of the information coming from variability for the study of our Galaxy, we focus here on what can be done with red giant stars and give a non-exhaustive list of breakthrough studies that could be conducted with a survey like Chronos (described in Section 4).

Generally speaking, understanding our Galaxy relies on a precise census of the positions, kinematics, chemical composition and age of its stars. The ongoing Gaia programme is building such a census with measurements of unprecedented precision for more than a billion stars over a large part of our Galaxy. The mass and age estimate however is presently the weak link of the chain. Most stellar masses and ages used in Galactic astronomy are still based on fitting stellar positions in Colour-Magnitude Diagrams (CMDs) with stellar evolution model isochrones (e.g., [69, 70]). The Gaia survey and the complementary large spectroscopic surveys will bring refined luminosity (L), effective temperature $\left(\mathrm{T}_{\text {eff }}\right)$, and chemical composition estimates to make this comparison. However, there are fundamental limitations to this approach:

- One limitation resides in the reliability of the theoretical evolution sequences used for comparison. This is a reason why improving our understanding of stellar structure and evolution is important and one of the ways to do it right now is stellar ensemble asteroseismology. This is why time domain surveys like CoRoT, Kepler, K2, TESS, or PLATO always represent an opportunity to improve the understanding of stellar structure and evolution and thus improve theoretical evolution sequences. This objective is included in the PLATO mission's scientific objectives and we can foresee great advances in the reliability of theoretical sequences for the main sequence cooler than early $\mathrm{F}$ stars and for the subsequent subgiant and red giant phase.

- The second limitation comes from the fact that, depending on the region considered in the HR diagram, $\mathrm{T}_{\text {eff }}$ and $\mathrm{L}$ values characterizing evolution sequences show more or less dependency on age, mass, and chemical composition. The case of red giants is very illustrative of this point. The red giant parts of the evolution sequences are clustering in a small range of $\mathrm{T}_{\text {eff. }}$ Gaia brings very precise measurement of $\mathrm{L}$ (thus of $\mathrm{R}$, for a known $\mathrm{T}_{\text {eff }}$ ), but the $\mathrm{T}_{\text {eff }}$ measurement, as precise as it can be, cannot bring a strong constraint on the mass estimate even if the chemical composition is assumed to be perfectly known. As a result, the constraint on the age is very poor, since for these red giants, mass is the predominant key factor for age on the RGB [71]. 
As commented in Section 2, one of the first pieces of information brought by seismology is the star's mean density. This is true for various types of pulsators and especially for red giants where the combination of the two seismic indices $\Delta v$ and $v_{\max }$ with an estimate of $\mathrm{T}_{\text {eff }}$ (which does not even have to be very precise, since the dependency in $\mathrm{T}_{\text {eff }}$ is small) leads straightforwardly to the mass estimate, which in turn provides a precise age estimate for several tens of thousands of red giants already.

A non-exhaustive list of breakthrough Galactic studies that could be conducted with a survey like Chronos in a few kpc around the Sun is the following:

- Chemical evolution of the Milky Way. Detailed relations between age - chemical abundance ratios of a variety of different elements (e.g., [72]) have proven to be fundamental for constraining the chemical evolution of our Galaxy, but we are just at the beginning of using them, because of the difficulty to obtain ages with the necessary precision. Yet, even with the precision provided by stellar isochrone age dating, these relations show remarkably tight correlations between age and chemical element abundance ratios that need to be confirmed with better ages, in particular for stars older than 8 Gyr (e.g., [73, 74, 75]). These relations in turn can be used to constrain the star formation history of the Milky Way, its gas accretion history, nucleosynthetic yields, as well as dynamical mechanisms such as stellar radial migration triggered by a combination of the effect of external and internal perturbations (such as the bar and spiral arms, e.g., [76]).

- Measuring the Milky Way's star formation history. The measurement of the Milky Way star formation history is not reflected in the age distribution of the stars in the solar vicinity, because most old stellar populations are confined to the inner Galaxy (e.g. [77]), beyond the reach of presently available stellar age dating methods. Today, our best prospect to measure the Star Formation History of our Galaxy is to measure the stellar mass density of populations as a function of alpha abundance, and to relate these abundances to a well-calibrated age scale, determined locally.

Ongoing or upcoming large spectroscopic surveys (see above) will provide deep spectroscopic sampling of all stellar populations throughout the Milky Way, to distances of several kpc, allowing the determination of stellar densities as a function of (alpha) abundances, or mono-abundance populations [77, 78]. Because of the relations linking age to alpha element abundances, chemical abundances can be used as a proxy for stellar age at very large distances, allowing us to determine the amount of stellar mass as a function of age in the Galaxy, or the Star Formation History. Ultimately, the validity of the determination of the Star Formation History will however depend on how accurately chemical abundances can be linked to an accurate age scale provided by the proposed survey. It will also allow us to better understand how the Milky Way disc was built (inside-out?) and crucially to compare the Milky Way history with other galaxies of the same mass.

- Understanding the early phases of our Galaxy. Precise ages, or high temporal resolution, are also necessary to understand the sequence of events that built the early Galaxy. Recent Gaia results suggest for instance that a large fraction of the oldest stars in the Galaxy have hot orbits aligned with the Galactic plane [3], and that the stellar halo is almost fully accreted (e.g., [4]). Moreover, it appears that these accretion events might in turn have played an important role in shaping the 
in-situ disc components of the Galaxy, in particular the thick disc component (e.g., [5]). The formation of the first stars, the rapid early chemical enrichment, the dissipation of the gas in a disc configuration, the accretion and merger of satellite galaxies, all these events occurred within a few Gyr, and a high temporal resolution, complemented with spectroscopic kinematic information is thus needed to disentangle the different components (in situ or accreted) and reconstruct the early history of our Galaxy. This can be done with Chronos with red giants in a few kpc around the Sun.

- Kinematics of the Galactic disc. At magnitude brighter than $\mathrm{G} \sim 12$, Gaia provides the best performance in terms of stellar radial velocities and proper motions, yielding extremely detailed kinematics of the solar vicinity $(\mathrm{d}<2-3 \mathrm{kpc}$ for red giants). While accretion events might have played an important role in the early shaping the in-situ disc components of the Galaxy, in particular the thick disc component (see above), it appears that more recent accretions are still influencing disc dynamics today. One of the main findings of Gaia has been a phase-spiral when looking at the phase-space surface of section of height vs. vertical velocity for local stars [6], which could not be revealed before due to uncertainties on parallaxes and proper motions. This clear out-of-equilbrium signature in the disc kinematics has been suggested to be the consequence of the passage of the Sagittarius Dwarf Galaxy through the plane of the Milky Way [6, 7], but other explanations based on the buckling of the Galactic bar have also been proposed [79], although the most likely explanation is a complex interplay between such external and internal mechanisms. What is certain is that it means that the hypothesis of equilibrium for modelling the Galactic disc can only be used as a basis for perturbation theory. A crucial observable allowing to disentangle the various proposed scenarios is the shape of this phase-spiral as a function of stellar ages (e.g., [7]), which Chronos will provide. Moreover, the current structure of the Galactic bar itself is also constrained from Gaia data on disc kinematics in the Solar vicinity (e.g., [9]), but again, the dynamical effects of non-axisymmetric perturbations on stellar populations of various ages would allow to disentangle better the effect of the bar and spiral arms [8], and also to constrain the age of the Galactic bar itself, which is still largely unknown.

The Chronos sample would thus allow a detailed study of the kinematics of the disc as a function of age, and would contribute to the understanding of the various effects of external and internal perturbations, such as resonances of the bar and spiral arms and their signature in the solar vicinity. Coupled with available spectroscopy, it would also permit the study of the effect of radial migration (e.g., [76]), for which the intensity and effect on stars in our neighbourhood is still largely debated (e.g., [80, 81]). More generally, a detailed characterization of the phase-space distribution of red giant stars as a function of age and chemical composition should help to reconstruct the global secular evolution of the Galaxy by making use of a dressed Fokker-Planck approach as presented in, e.g., Fouvry et al. [82].

The above four examples of ground-breaking studies in the context of Galactic dynamics and archaeology are limited to red giants for which we already have developed a sufficient understanding to build precise seismic diagnostics and seismic characterization. Beyond red giants, as we explain in Section 2, we can foresee a 
natural extension of the application of seismic characterization on large portions of the sky to main sequence solar-type pulsators (with PLATO) and to very luminous AGB stars (with the Rubin Observatory). We also mentioned in Section 2 the interest and possibility to extend this approach to main sequence classical pulsators. Because their pulsations are not so different in characteristic time scales, in amplitude and in necessary resolution frequency, these objects would also be covered by the survey considered in Section 4.

Applying seismic characterization to pulsating stars with mass between $1.5 \mathrm{M}$ and $15 \mathrm{M}$ on the main sequence and subgiant branch would enrich the study of our Galactic neighbourhood (not as deep as for red giants which are intrinsically very luminous, but on a few hundreds of pc) and open new perspective also because of the much shorter time scales of evolution toward high masses.

\section{Chronos - An all-sky long-term seismic survey to characterize half a million red giant stars up to $1.7 \mathrm{kpc}$ around the sun}

Here we show that it is possible to plan an all-sky, fast cadence, long duration photometric survey satisfying the needs of the scientific objective introduced in Section 3. The concept presented in Section 4.1 is of course only illustrative and possible ameliorations are left aside for a later phase. As it is, we would expect this project to match the requirements for a medium size ESA mission. In Section 4.2, Chronos main characteristic features are compared with relevant existing and planned missions.

\subsection{A possible instrument and mission profile}

In order to reach long duration observations and all-sky coverage, the proposed mission relies on two main features: an instrument mounted on a spinning platform and a suitable observational strategy. They are described respectively in Section 4.1.1 and Section 4.1.2 hereafter (see also Table 1). Performances in terms of seismic diagnostics are given in Table 2 .

\subsubsection{Mission concept - the instrument and the spinning platform}

A platform carries eight identical elementary cameras, each covering a $11.25 \times$ $11.25 \mathrm{deg}^{2}$ field of view (FOV). Cameras are grouped by two, so that at any time they are staring at four fields on the sky, $22.5 \times 11.25 \mathrm{deg}^{2}$ each (see Fig.1).

The platform is spinning (Fig. 1) around the same rotational axis during a continuous duration Dc (here we take Dc $=3.75$ months) and the detectors "sweep" regularly a $22.5 \times 360 \mathrm{deg}^{2}$ corona on the sky. The rotation period $\mathrm{P}_{\text {rot }}$ (here $\mathrm{P}_{\text {rot }}=8 \mathrm{~min}$ ) ensures that each star of this corona is observed regularly with a sampling time Ts $=\mathrm{P}_{\text {rot }} / 4$ (here $\mathrm{Ts}=2 \mathrm{~min}$ ).

The image of each star crosses the focal plane in $15 \mathrm{~s}$ and thus one CCD in $7.5 \mathrm{~s}$. The CCDs are read in TDI mode (time delay integration mode, as for Gaia, where images cross the CCD in $4.5 \mathrm{~s}$ ), the charges being drifted at the same speed as the star images.

A first optical sketch has been drawn considering, for each camera, a dioptric (6lenses) system with a $30 \mathrm{~cm}$ objective, illuminating a focal plane with four CCDs 

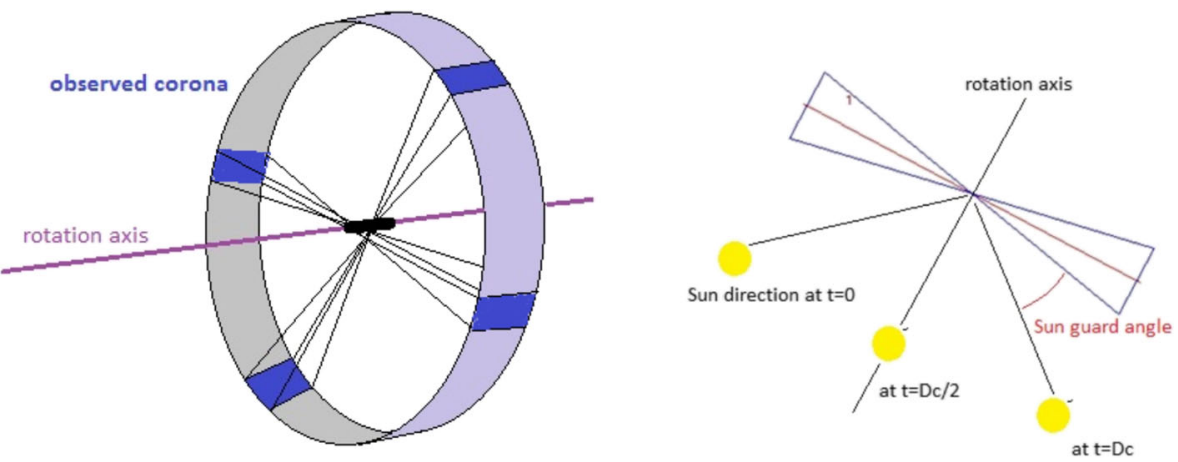

Fig. 1. left: The $22.5 \times 360 \mathrm{deg}^{2}$ sky corona swept by the instrument every $\mathrm{Ts}=2 \mathrm{~min}$ (rotation period of the platform $8 \mathrm{~min}$ ). Right: the same corona is observed during Dc, keeping the Sun away by the guard angle (GA).

(4.5 k $\times 4.5 \mathrm{k}$ pixels, $18 \mu \mathrm{m} / \mathrm{px}$, similar to PLATO CCDs) and a point spread function within 4 pixels anywhere on the square FOV $11.25 \times 11.25 \mathrm{deg}^{2}$ (Tables 1$)$.

\subsubsection{Mission concept - orbit and observational strategy}

We considered a platform located at Sun-Earth L2, observing the same corona on the sky during $\mathrm{Dc}=3.75$ months and change from one corona to the next. With a rotation axis chosen to point toward the Sun at Dc/2, the Sun is kept away from the FOV by a guard angle GA (here GA = $22.5 \mathrm{deg}$ ). For the different phases described here after, performances in terms of seismic diagnostics are summarized in Table 2.

Phase 1 (2.5 years): by shifting every Dc (3.75 months) the rotation axis by $112.5 \mathrm{deg}(112.5=3.75 \times 30$ being the angle described by the Sun in 3.75 months $)$, it is possible to cover the whole sky with 8 successive coronas within 30 months. For comparison, covering the whole sky by shifting every 3 months a pointed instrument with a FOV of $1000 \mathrm{deg}^{2}$ requires more than 10 years.

Table 1 Summary of the main figures of the mission concept in its present version

\begin{tabular}{ll}
\hline Chronos main figures & Sizing \\
\hline Entrance pupil & $30 \mathrm{~cm}$ \\
Effective pupil* & $10.6 \mathrm{~cm}$ \\
Number of detection blocks & 4 \\
Number of cameras / block & 2 \\
Observation cadence & $2 \mathrm{~min}$ \\
FOV of a camera & $11.25^{\circ}$ \\
Size of each corona field & $22.5^{\circ} \times 360^{\circ}$ \\
Observation shift between consecutive corona & $112.5^{\circ}$ \\
Observation length per corona & 3.75 months $(2$ times in nominal lifetime $)$ \\
Mission nominal lifetime & 5 years \\
\hline
\end{tabular}

*The effective pupil takes into account that each star of a given corona is observed by a camera regularly but during $1 / 8$ of the time 
Table 2 Limiting $\mathrm{m}_{\mathrm{V}}$ magnitude for $\Delta v$ and $v_{\max }$ measurement, for different observation durations and different regions of the HR diagram [83].

\begin{tabular}{|l|l|l|l|l|l|l|l|}
\hline \multicolumn{2}{|l|}{ Observation duration } & $\begin{array}{l}\text { MS } \\
\text { type G }\end{array}$ & $\begin{array}{l}\text { MS } \\
\text { type F }\end{array}$ & Subgiant & Early RGB & Red clump & Early AGB \\
\hline \multirow{3}{*}{3.5 months } & $1 \mathrm{x}$ & 8.0 & 8.8 & 9.5 & 10.5 & 12.0 & 11.1 \\
\cline { 2 - 8 } & $2 \mathrm{x}$ & 8.6 & 9.5 & 10.2 & 11.0 & 13.3 & 12.4 \\
\cline { 2 - 8 } & $3 \mathrm{x}$ & 9.2 & 10.2 & 10.8 & 11.5 & 14.6 & 13.7 \\
\hline 5 years & $\mathrm{CVZ}$ & 11.3 & 12.4 & 12.9 & 13.5 & 16.0 & 15.1 \\
\hline
\end{tabular}

Green indicates the matching with the $8-11 \mathrm{~m}_{\mathrm{V}}$ range observable with Chronos in its present version.

We notice that beside this, the two regions of the sky where the 8 coronas overlap will be observed a longer time (up to the total length of the mission for the Continuous Viewing Zone (CVZ), two symmetric discs of diameter 22.5 deg centred at the ecliptic poles).

Phase 2 ( +30 months $\rightarrow 5$ years, nominal duration of the mission): with 16 runs of duration Dc, the whole sky will be observed twice and each target observed two times Dc, separated by 7 Dc (here $2 \times 3.75$ months separated by $\sim 2$ years and 2 months each).

Phase 3 (possible extension: $+\mathbf{3 0}$ months $\rightarrow 7$ years and 6 months): with 24 runs of duration Dc, the whole sky will be observed 3 times Dc, separated by 7 Dc (here $3 \times$ 3.75 months separated by $\sim 2$ years and 2 months each).

The continuous viewing zone The survey of Chronos gives access to a Continuous Viewing Zone (CVZ), two cones of angular diameter $22.5 \mathrm{deg}$, about the size of the

Table 3 Comparison of Chronos' main figures with other past and expected projects. For the various projects, estimates are obtained from the information available in the references mentioned in the text and at the respective projects' web pages

\begin{tabular}{|c|c|c|c|c|c|}
\hline \multirow[t]{2}{*}{ Project } & \multirow[t]{2}{*}{ Sky coverage $(\%)$} & \multicolumn{2}{|c|}{ Observation length and sky fraction } & \multicolumn{2}{|c|}{$\begin{array}{l}\text { Cadence of observation and } \\
\text { number of observed stars }\end{array}$} \\
\hline & & Min & Max & $\mathrm{SC}$ & $\mathrm{LC}$ \\
\hline CoRoT & 0.20 & 1 month & 5 months & $\begin{array}{l}32 \mathrm{~s} \\
\sim 1 \mathrm{k}\end{array}$ & $\begin{array}{l}8.5 \min \\
\sim 150 \mathrm{k}\end{array}$ \\
\hline Kepler & 0.25 & 1 month & 4 years & $\begin{array}{l}2 \min \\
\sim 1 \mathrm{k}\end{array}$ & $\begin{array}{l}30 \mathrm{~min} \\
\sim 150 \mathrm{k}\end{array}$ \\
\hline $\mathrm{K} 2$ & 2.5 & 80 days & & $\sim 5 \mathrm{k}$ & $\sim 2 \mathrm{M}$ \\
\hline TESS & 86 & $\begin{array}{l}27 \text { days }(63 \%) \\
54 \text { days }(23 \%)\end{array}$ & 1 year $(\mathrm{CVZ} \sim 2 \%)$ & $\begin{array}{l}2 \min \\
\sim 200 \mathrm{k}\end{array}$ & $\begin{array}{l}30 \mathrm{~min} \\
\sim 1 \mathrm{M}\end{array}$ \\
\hline Rubin Observatory & $\sim 50$ & 10 years & & $\sim 3$ days & \\
\hline PLATO & $\sim 50$ & 2 months $(50 \%)$ & 3 years $(10 \%)$ & $1 \mathrm{~min}$ & $\begin{array}{l}10 \mathrm{~min} \\
\sim 1 \mathrm{M}\end{array}$ \\
\hline Chronos & 100 & $\begin{array}{l}2 \times 3.75 \text { months } \\
(100 \%)\end{array}$ & $\begin{array}{l}>5 \text { years } \\
(\mathrm{CVZ} \sim 2 \%)\end{array}$ & $\begin{array}{l}2 \mathrm{~min} \\
1 \mathrm{M}\end{array}$ & \\
\hline
\end{tabular}


TESS CVZ (24 deg) and in the same region as TESS (Ecliptic poles). The scientific yield for stars observed 5 years in the CVZ will surpass all projects, with many more objects to be observed much longer in all regions of the HR diagram. For these stars, the most precise seismic analysis will be performed: analysis of the signatures of helium, rotation, and convection for main sequence stars; core properties and rotation profiles for evolved stars.

\subsection{Programmatic context: Comparison with existing and planned space and ground based projects}

As mentioned previously, the need for photometric surveys covering a large part of the sky over long durations has been taken into account as much as possible in various existing and planned space and ground-based projects. However, there are strong practical difficulties to optimize observation for such a large range of contradictory constraints. We show here that none of these projects is suited for the objectives identified in our present scientific proposal (see Table 3). This is understandable since these projects usually had to optimize in priority other scientific objectives with other instrumental constraints, such as the search for exoplanets.

CoRoT, Kepler and K2 CoRoT and Kepler have brought wonderful data for red giant stars, because the duration of the runs was at least several months and up to years in the case of Kepler. But this long duration was paid in both cases by a limited coverage of the sky. The K2 extension of the Kepler mission allowed to enlarge the sky coverage considerably, (although it was still not complete) but the duration of the runs was limited to 80 days, which is not enough to derive the seismic indices needed in the present project.

Tess With $86 \%$ of all the sky accessible, TESS [84] is the project offering presently the largest sky coverage. However, the primary goal of TESS is to discover planets smaller than Neptune that transit stars bright enough to enable follow-up spectroscopic observations that can provide planet masses and atmospheric compositions, with short revolution periods and most of the sky (63\%) will be observed for only 27 days within the 2-year nominal mission and only $23 \%$ will be observed for 54 days or more. This duration is too short to measure seismic indices for red giant stars.

Plato The PLATO mission profile [85] is organized around 2 long duration fields totalling $4400 \mathrm{deg}^{2}$ to be observed during 2 to 3 years each. Then, it is planned to complete the observations with a few step-and-stare fields of shorter duration in order to cover about half the sky. Considering the scientific objective considered here, the two long duration fields will bring valuable data for red giants, but on a limited fraction of the sky. The step-and-stare fields, if they have to increase this sky coverage significantly, say to half the sky, will hardly reach more than 2.5 months each. This would be a great survey in terms of solar-type main sequence pulsators for which the sample of data available is still limited [86,87], but this duration is too short to properly estimate seismic indices like $\Delta \mathrm{P}$ for red giants. 
Rubin observatory (previously known as LSST) The Rubin Observatory survey [88] will cover about half the sky (southern hemisphere) with two consecutive $15 \mathrm{~s}$ measurements every 3 days at best. Such a sampling rate is not suited for the red giant stars considered in the present project. On the other hand, it will be very efficient for AGB stars or very large red giants near the tip of the red giant branch, which require very long duration observations (7-10 years) as planned for the Rubin Observatory.

Chronos The present project, offering an all-sky survey with long duration $(2 \times$ 3.75 months within the 5-year nominal mission) will bridge the gap left between the Rubin Observatory and PLATO, bringing seismic indices for solar-type pulsators from subgiants to early AGB stages ( $\sim$ half a million red giants and subgiants), plus classical pulsators on the main sequence ( $\delta$ Scuti, $\gamma$ Doradus, $\beta$ Cephei, and Slowly Pulsating Btype (SPB) stars).

\section{Possible instrumental improvements and technology challenges}

The technology considered here to support the Chronos Project is inherited from the various photometric surveys realized or designed over the last decades (CoRoT, Kepler, TESS, PLATO) and from the Gaia mission for the spinning platform and the time delay integration (TDI) readout of the CCDs.

We thus are reasonably confident in the possibility to reach the performances anticipated, even if there is probably room to optimize the instrumental concept considered here.

\subsection{An orbit allowing longer individual continuous runs (beyond 3.75 months)}

One interesting possible significant improvement would be to find an orbit allowing uninterrupted observations longer than the 3.75 months considered in the present version. Compared with the present $2 \times 3.75$ months sequences, an uninterrupted sequence would allow not only to measure $\Delta \mathrm{P}$ but also $\Delta \Pi 1$, the asymptotic period spacing which is a very sensitive indicator for evolution but requires the measurement of g-dominated mixed modes with long lifetime. These modes appear as coherent pulsations and are unresolved in the Fourier spectrum even for long durations of several years. In such a case, continuous runs of e.g. 7.5 months would be better than discontinuous $(2 \times 3.75$ months $)$.

Increasing the duration would require a change in the present concept that is challenging. Among possibilities we could think of:

- an orbit eclipsing the Sun: the length of individual runs DC* (Dc* = 3.75 months in the present setup) is determined by the will not to have the Sun coming closer than GA $=22.5 \mathrm{deg}$ from the observed corona. This constraint could be relaxed (and $\mathrm{Dc}^{*}$ increased without limitation) if it were possible to operate an orbit where the platform is in the shadow of the Earth (or of an artificial shield) at least during the 1-2 months when the Sun crosses the corona (possibly all the time). 
- positioning the platform further from the Sun. For instance, at Mars L2 point (or anywhere at the distance of Mars from the Sun): this would naturally increase Dc* by a factor $\sim 2$ (year_Mars/Year_Earth $=1.9$ ) without changing the mission observational strategy.

\subsection{An adaptation of the TDI mode allowing an increase of the angular size of the coronas}

In the present design, coronas are limited to 22.5 deg width. Enlarging this width (on the side opposite to the Sun) would improve the sky coverage performances. This increase however would lead star images to describe curved trajectories on the CCDs instead of staying in the same $\sim 4$ columns. The solution consisting in increasing the number of columns considered for each star is a possibility but rapidly becomes unsatisfying because of the overlap of the images of different stars.

A possibility could be:

- adapt the TDI readout mode to take into account the curvature of the trajectory of star images when crossing the CCD. This would mean attributing to each star the photoelectrons collected in different columns as time varies. This approach has to be tested, but the fact that we stay on one given corona for several months should help since the trajectories are not expected to change at first order. We note that this option would also relax the constraints on the alignment of the CCDs ( 1 ' in the present design to keep the trajectory within the same 5 columns) and on the stability of the orientation of the rotation axis (also 1' in the present design).

\section{Summary and conclusion in a broad scientific perspective}

The period considered for the ESA Voyage 2035-2050 long-term plan lies at the crossroad of several projects bringing major progress in the characterization of the stellar content of our Galaxy. Gaia and the various spectroscopic large surveys are building up an unprecedented census in terms of astrometric, kinematic, and chemical properties of the Galactic stellar populations. Within a decade, precise measurements of these properties will thus be available for hundreds of millions of stars over a large part of our Galaxy. Meanwhile, stellar time domain surveys initiated by CoRoT and Kepler and continued by space missions like TESS and PLATO or ground-based projects like the Rubin Observatory will have brought stellar seismology and stellar seismic characterization to a high level of maturity. The synergy between seismic information and astrometric information and the great interest for Galactic population studies, have already been illustrated with tens of thousands of red giant stars and we can foresee its extension to other types of stars. This is why we are convinced that an all-sky, high-cadence, long-duration variability survey will be a top scientific priority in the $2035-50$ period.

The Chronos concept presented here, constitutes a time-domain extension of Gaia. It will allow mass and age estimates for half a million red giants within $1.7 \mathrm{kpc}$ from the Sun and shed a new light on our understanding of the Galactic dynamics and archaeology. 
In terms of characteristic time scales, Chronos will bridge the gap between PLATO and the Rubin Observatory. It will surpass all previous time-domain projects in terms of sky coverage $(100 \%)$ and duration $(2 \times 3.75$ months within the 5-year nominal mission).

Open Access This article is licensed under a Creative Commons Attribution 4.0 International License, which permits use, sharing, adaptation, distribution and reproduction in any medium or format, as long as you give appropriate credit to the original author(s) and the source, provide a link to the Creative Commons licence, and indicate if changes were made. The images or other third party material in this article are included in the article's Creative Commons licence, unless indicated otherwise in a credit line to the material. If material is not included in the article's Creative Commons licence and your intended use is not permitted by statutory regulation or exceeds the permitted use, you will need to obtain permission directly from the copyright holder. To view a copy of this licence, visit http://creativecommons.org/licenses/by/4.0/.

\section{References}

1. Gaia Collaboration, et al.: Gaia Data Release 2. Summary of the contents and survey properties. A\&A. 616, A1 (2018a)

2. Gaia Collaboration, et al.: Gaia Data Release 2. Kinematics of globular clusters and dwarf galaxies around the Milky Way. A\&A. 616, A12 (2018b)

3. Sestito, F.: Tracing the formation of the milky way through ultra metal-poor stars. MNRAS. 484, 2166 (2019)

4. Di Matteo, P., et al.: The Milky Way has no in-situ halo but it has a thick disc. Composition of the stellar halo and age-dating the last significant merger with Gaia DR2 and APOGEE. arXiv:1812.08232 (2018)

5. Helmi, A., et al.: The merger that led to the formation of the milky Way's inner stellar halo and thick disk. Nature. 563, 85 (2018)

6. Antoja, T., et al.: A dynamically young and perturbed milky way disk. Nature. 561, 360 (2018)

7. Laporte, C., et al.: Footprints of the Sagittarius dwarf galaxy in the Gaia data set. MNRAS. 485, 3134 (2019)

8. Laporte, C., et al.: Bar resonances and low angular momentum moving groups in the Galaxy revealed by their stellar ages. A\&A. 643, 3 (2020). https://doi.org/10.1051/0004-6361/202038740

9. Monari, G., et al.: Signatures of the resonances of a large galactic bar in local velocity space. A\&A. 626, A41 (2019)

10. Baglin, A., et al.: CoRoT: a high precision photometer for stellar evolution and exoplanet finding. in 36th COSPAR Scientific Assembly. COSPAR Meeting. 36, 3749 (2006)

11. Borucki, W.J., et al.: Kepler planet-detection Mission: introduction and first results. Science. 327, 977 (2010)

12. Bedding, T., et al.: Gravity modes as a way to distinguish between hydrogen- and helium -burning red giant stars. Nature. 471, 608 (2011)

13. Belkacem, K.: Determination of the stars fundamental parameters using seismic scaling relations. Proc. SF2A 2012, 173-188 arXiv:1210.3505 (2012)

14. Kallinger, T., et al.: Oscillating red giants in the CoRoT exofield: asteroseismic mass and radius determination. A\&A. 509, 77 (2010). https://doi.org/10.1051/0004-6361/200811437

15. Mosser, B., Appourchaux, T.: On detecting the large separation in the autocorrelation of stellar oscillation times series. A\&A. 508, 877 (2009). https://doi.org/10.1051/0004-6361/200912944

16. Mosser, B., et al.: Probing the core structure and evolution of red giants using gravity-dominated mixed modes observed with Kepler. A\&A. 540, A143 (2012a)

17. Mosser, B., et al.: Spin down of the core rotation in red giants. A\&A. 548, A10 (2012b)

18. de Ridder, J., et al.: Non-radial oscillation modes with long lifetimes in giant stars. Nature. 459, 398 (2009)

19. Eddington, A.S.: On the pulsations of a gaseous star. MNRAS. 79, 177 (1919)

20. Belkacem, K., et al.: The underlying physical meaning of the vmax - vc relation. A\&A. 530, A142 (2011)

21. Bruntt, H., et al.: Accurate fundamental parameters and detailed abundance patterns from spectroscopy of 93 solar-type Kepler targets. MNRAS. 423, 122 (2012)

22. De Assis Peralta, R., et al.: A new method for extracting seismic indices and granulation parameters: results for more than 20,000 CoRoT and Kepler red giants. AN. 339, 134 (2018)

23. Pande, D., et al.: Surface gravities for 15000 Kepler stars measured from stellar granulation and validated with Gaia DR2 parallaxes. MNRAS. 480, 467 (2018) 
24. Yu, J., et al.: Asteroseismology of 16,000 Kepler Red Giants: Global Oscillation Parameters, Masses, and Radii. ApJS. 236, 42 (2018). https://doi.org/10.3847/1538-4365/aaaf74

25. Vrard, M., et al.: Period spacings in red giants. II. Automated measurement. A\&A. 588, A87 (2016)

26. Montalbán, J., et al.: Testing convective-core overshooting using period Spacings of dipole modes in red giants. ApJ. 766, 118 (2013)

27. Mosser, B., et al.: The universal red-giant oscillation pattern. An automated determination with CoRoT data. A\&A. 525, L9 (2011). https://doi.org/10.1051/0004-6361/201015440

28. Beck, P., et al.: Fast core-rotation in red-giant stars as revealed by gravity-dominated mixed modes. Nature. 481, 55 (2012)

29. Deheuvels, S., et al.: Seismic evidence for a rapidly rotating Core in a lower-giant-branch star observed with Kepler. ApJ. 756, 19 (2014)

30. Géhan, C., et al.: Core rotation braking on the red giant branch for various mass ranges. A\&A. 616, A24 (2018)

31. Vrard, M., et al.: Helium signature in red giant oscillation patterns observed by Kepler. A\&A. 579, A84 (2015)

32. Vrard, M., et al.: Amplitude and lifetime of radial modes in red giant star spectra observed by Kepler. A\&A. 616, A94 (2018)

33. Khan, S., et al.: The Red-giant Branch Bump Revisited: Constraints on Envelope Overshooting in a Wide Range of Masses and Metallicities. ApJ. 859, 156 (2018). https://doi.org/10.3847/1538-4357/aabf90

34. Breger, M., et al.: Regularities in frequency spacings of $\delta$ Scuti stars: the Kepler star KIC 9700322. MNRAS. 414, 1721 (2011)

35. Garcia Hernández, A., et al.: An in-depth study of HD 174966 with CoRoT photometry and HARPS spectroscopy. Large separation as a new observable for $\delta$ Scuti stars. A\&A. 559, A63 (2013)

36. Michel, E., et al.: What CoRoT tells us about delta Scuti stars - existence of a regular pattern and seismic indices to characterize stars. EPJ Web of Conferences. 160, 03001 (2017)

37. Paparo, M., et al.: Unexpected series of regular frequency spacing of $\delta$ Scuti stars in the non-asymptotic regime. II. Sample-Echelle Diagrams and Rotation. ApJS. 224, 41 (2016)

38. Garcia Hernández, A., et al.: Observational $\Delta v-\bar{\rho}$ relation for $\delta$ Sct stars using eclipsing binaries and space photometry. ApJ. 811L, 29 (2015)

39. García Hernández, A., et al.: Precise surface gravities of $\delta$ Scuti stars from asteroseismology. MNRAS. 471, 140 (2017)

40. Bravo, J.P., et al.: Wavelets: a powerful tool for studying rotation, activity, and pulsation in Kepler and CoRoT stellar light curves. A\&A. 568, A34 (2014)

41. De Medeiros, J.R., et al.: Overview of semi-sinusoidal stellar variability with the CoRoT satellite. A\&A. 555, A63 (2013)

42. Garcia, R.A., et al.: Measuring reliable surface rotation rates from Kepler photometric observations. ASP Conference Series. 479, 129 (2013)

43. Lanza, A., et al.: Photospheric activity, rotation, and radial velocity variations of the planet-hosting star CoRoT-7. A\&A. 520, A53 (2010)

44. Leão, I.C., et al.: Rotation period distribution of CoRoT_and Kepler sun-like stars. A\&A. 582, A85 (2015)

45. Balona, L.: Evidence for spots on hot stars requires major revision of stellar physics. MNRAS. 490, 2112 (2019). https://doi.org/10.1093/mnras/stz2808

46. David-Uraz, A., et al.: Magnetic OB[a] stars with TESS: probing their evolutionary and rotational properties (MOBSTER) - I. First-light observations of known magnetic B and A stars. MNRAS. 487, 304 (2019)

47. Savanov, I.S.: Spots and activity of A-type stars. ASPC. 518, 131 (2019)

48. Blazere, A., et al.: Detection of ultra-weak magnetic fields in am stars: $\beta$ Ursae Majoris and $\theta$ Leonis. A\&A. 586A, 97 (2016)

49. Böhm, T., et al.: Discovery of starspots on Vega. First spectroscopic detection of surface structures on a normal A-type star. A\&A. 577A, 64 (2015)

50. Lignières, F., et al.: First evidence of a magnetic field on Vega. Towards a new class of magnetic A-type stars. A\&A. 500L(41), (2009)

51. Barnes, S.A.: Ages for illustrative field stars using Gyrochronology: viability, limitations, and errors. ApJ. 669, 1167 (2007)

52. Meibom, S., et al.: The Kepler cluster study: stellar rotation in NGC 6811. ApJ. 733, L9 (2011)

53. Bastien, F.A., et al.: An observational correlation between stellar brightness variations and surface gravity. Nature. 500, 427 (2013). https://doi.org/10.1038/nature12419

54. Kallinger, T., et al.: Precise stellar surface gravities from the time scales of convectively driven brightness variations. Sci. Adv. 2, 15006 (2016)

55. Mathur, S., et al.: Granulation in Red Giants: Observations by the Kepler Mission and Three-dimensional Convection Simulations. ApJ. 741, 119 (2011). https://doi.org/10.1088/0004-637X/741/2/119 
56. Mosser, B., et al.: Period-luminosity relations in evolved red giants explained by solar-like oscillations. A\&A. 559, 137 (2013)

57. Soszynski, I., et al.: The optical gravitational lensing experiment. Small Amplitude Variable Red Giants in the Magellanic Clouds. Acta Astron. 54, 129 (2004)

58. Soszynski, I., et al.: The optical gravitational lensing experiment. The OGLE-III Catalog of Variable Stars. IV. Long-Period Variables in the Large Magellanic Cloud. Acta Astron. 59, 239 (2009)

59. Takayama, M., et al.: On the pulsation modes of OGLE small amplitude red giant variables in the LMC. MNRAS. 431, 3189 (2013)

60. Majewski, S., et al.: The apache point observatory galactic evolution experiment (APOGEE). AJ. 154, 94 (2017)

61. Dalton, G., et al.: WEAVE: the next generation wide-field spectroscopy facility for the William Herschel Telescope. SPIE, 8446, id. 84460P (2012)

62. De Jong, R.S., et al.: 4MOST: project overview and information for the first call for proposals. Messenger. 175, 3 (2019). https://doi.org/10.18727/0722-6691/5117

63. Anders, F., et al.: Galactic archaeology with asteroseismology and spectroscopy: Red giants observed by CoRoT and APOGEE. A\&A. 597, 30 (2017a). https://doi.org/10.1051/0004-6361/201527204

64. Anders, F., et al.: Red giants observed by CoRoT and APOGEE: The evolution of the Milky Way's radial metallicity gradient. A\&A. 600, 70 (2017b). https://doi.org/10.1051/0004-6361/201629363

65. Miglio, A., et al.: Probing populations of red giants in the galactic disk with CoRoT. A\&A. 503, L21 (2009)

66. Miglio, A., et al.: Galactic archaeology: mapping and dating stellar populations with asteroseismology of red-giant stars. MNRAS. 429, 423 (2013)

67. Rendle, B.M., et al.: The K2 galactic caps project - going beyond the Kepler field and ageing the galactic disc. MNRAS. 490, 4465 (2019). https://doi.org/10.1093/mnras/stz2454

68. Valentini, M., et al.: Masses and ages for metal-poor stars. A pilot programme combining asteroseismology and high-resolution spectroscopic follow-up of RAVE halo stars. A\&A. 627, 173 (2019). https://doi.org/10.1051/0004-6361/201834081

69. Gallart, C., et al.: Uncovering the Birth of the Milky Way through Accurate Stellar Ages with Gaia. Nature Astronomy, Advanced Online Publication (2019)

70. Sanders, J., et al.: Isochrone ages for $\tilde{3}$ million stars with the second Gaia data release. MNRAS. 481, 4093 (2018)

71. Miglio, A., et al.: PLATO as it is : a legacy mission for galactic archaeology. AN. 338, 644 (2017)

72. Snaith, O., et al.: Reconstructing the star formation history of the Milky Way disc(s) from chemical abundances. A\&A. 578, A87 (2015). https://doi.org/10.1051/0004-6361/201424281

73. Haywood, M., et al.: The age structure of stellar populations in the solar vicinity. Clues of a two-phase formation history of the Milky Way disk. A\&A. 560, 109 (2013)

74. Nissen, P.E., et al.: High-precision abundances of elements in Kepler LEGACY stars. Verification of trends with stellar age. A\&A. 608, 112 (2017)

75. Spina, L., et al.: Nucleosynthetic history of elements in the Galactic disk. [X/Fe]-age relations from highprecision spectroscopy. A\&A. 593, A125 (2016). https://doi.org/10.1051/0004-6361/201628557

76. Minchev, I., Famaey, B.: A New Mechanism for Radial Migration in Galactic Disks: Spiral-Bar Resonance Overlap. ApJ. 722, 112 (2010). https://doi.org/10.1088/0004-637X/722/1/112

77. Bovy, J., et al.: The Spatial Structure of Mono-abundance Sub-populations of the Milky Way Disk. ApJ. 753, 148 (2012). https://doi.org/10.1088/0004-637X/753/2/148

78. Mackereth, T., et al.: The age-metallicity structure of the milky way disc using APOGEE. MNRAS. 471, 3057 (2017)

79. Khoperskov, S., et al.: The echo of the bar buckling: phase-space spirals in Gaia data release 2. A\&A. 622, L6 (2019)

80. Hallé, A., et al.: Quantifying stellar radial migration in an N-body simulation: blurring, churning, and the outer regions of galaxy discs. A\&A. 578, 58 (2015)

81. Minchev, I., et al.: Radial migration in galactic disks caused by resonance overlap of multiple patterns: self-consistent simulations. A\&A. 527, A147 (2011)

82. Fouvry, J.-B., et al.: Self-gravity, resonances, and orbital diffusion in stellar disks. ApJ. 806, 117 (2015)

83. Mosser, B., et al.: Seismic performance. A\&A. 622, 76 (2019). https://doi.org/10.1051/0004-6361/ 201834607

84. Ricker, G.R., et al.: Transiting Exoplanet Survey Satellite (TESS). JATIS...1a4003R (2015). https://doi. org/10.1117/1.JATIS.1.1.014003

85. Rauer, H., et al.: The PLATO mission. AN. 337, 961 (2016)

86. Chaplin, W., et al.: The Asteroseismic potential of Kepler: first results for solar-type stars. ApJL. 713, L169 (2010) 
87. Silva Aguirre, V., et al.: Verifying Asteroseismically determined parameters of Kepler stars using Hipparcos parallaxes: self-consistent stellar properties and distances. ApJ. 757, 99 (2012)

88. LSST Science Collaboration, et al.: LSST Science book. arXiv:0912.0201 (2009)

Publisher's note Springer Nature remains neutral with regard to jurisdictional claims in published maps and institutional affiliations.

\section{Affiliations}

Eric Michel ${ }^{1} \cdot$ Misha Haywood $^{2} \cdot$ Benoit Famaey $^{3} \cdot$ Benoit Mosser $^{1}$. Reza Samadi $^{1}$ • Mario J.P.F.G. Monteiro ${ }^{4,5} \cdot$ Hans Kjeldsen $^{6} \cdot$ Kevin Belkacem $^{1}$. Andréa Miglio $^{7}$ - Rafael Garcia ${ }^{8}$. David Katz ${ }^{9}$. Juan Carlos Suarez ${ }^{10}$. Sébastien Deheuvels ${ }^{11}$. Tiago Campante ${ }^{3,4}$. Margarida Cunha ${ }^{4,5}$. Victor Silva Aguirre ${ }^{6}$ • Jerôme Ballot ${ }^{11}$. Andy Moya ${ }^{12}$

Eric Michel

Eric.Michel@obspm.fr

$\triangle$ Andréa Miglio

andrea.miglio@unibo.it

1 LESIA, Observatoire de Paris, Université PSL, CNRS, Sorbonne Université, Université de Paris, Paris, France

2 GEPI, Observatoire de Paris, Université PSL, CNRS, Paris, France

3 Université de Strasbourg, CNRS, UMR 7550, Observatoire astronomique de Strasbourg, 11 rue de l'Université, 67000 Strasbourg, France

4 Instituto de Astrofísica e Ciências do Espaço, Universidade do Porto, Rua das Estrelas, 4150-762 Porto, Portugal

5 Departamento de Física e Astronomia, Faculdade de Ciências da Universidade do Porto, Rua do Campo Alegre, s/n, 4169-007 Porto, Portugal

6 Stellar Astrophysics Centre, Department of Physics and Astronomy, Aarhus University, Aarhus, Denmark

7 University of Birmingham, Birmingham, UK

8 Département d'Astrophysique, IRFU/DRF/CEA Saclay, L'Orme des Merisiers, bat. 709, 91191 Gif-sur-Yvette Cedex, France

9 GEPI, Observatoire de Paris, Université PSL, CNRS, Paris, France

10 Dept. Física Teórica y del Cosmos Dept. University of Granada Facultad de Ciencias, 18071 Granada, Spain

11 IRAP, Université de Toulouse, CNRS, CNES, UPS, Toulouse, France

12 Electrical Engineering, Electronics, Automation and applied Physics Department, E.T.S.I.D.I., Polytechnic University of Madrid, 28012 Madrid, Spain 